\title{
Robotic Thyroidectomy and Infection
}

Sevgi Buyukbese Sarsu', Hasan Ucmak², Mehmet Akif Buyukbese²

\section{thection}

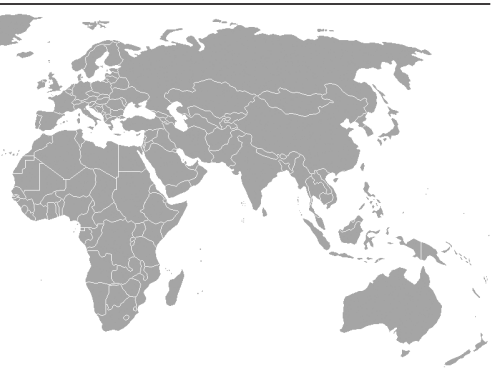

Oncoplastic surgery is constantly improved because of increasing rates of thyroid cancer and requirement of a good cosmesis in those patients in order to eliminate a large neck scar. Bilateral axillo-breast approach (BABA) provides a symmetric and good view during the procedure. Follow-up studies show that permanent hypoparathyroidism (hypocalcemia) and laryngeal nerve damage (hoarseness) may be observed as postoperative complications.

In one study, 704 patients received BABA robot-assisted endoscopic thyroidectomy (RoT): $7.8 \%$ total thyroidectomy, $66.3 \%$ total thyroidectomy + central neck dissection, $4.8 \%$ total thyroidectomy + central and lateral neck dissection, 9.5\% subtotal thyroidectomy, and $10.4 \%$ lobectomy. There was no infectious complication among those patients (1). Between 2008-2009, 41 patients received RoT because of thyroid nodules. Unilateral axillo-breast or axillary approach was used. No infection complication was found (2). Between the same year period in a case series of 302 patients, 138 open and 69 BABA RoT were performed. Again, no infection complication was observed (3). In another study, 109 patients underwent robotic total thyroidectomy because of papillary thyroid cancer. BABA method was preferred. No complication was determined (4). Between 2007-2009, 1043 patients (71 male and 972 female) with low-risk differentiated thyroid carcinoma received 366 total thyroidectomy and 677 subtotal thyroidectomy by robotic method using transaxillary approach performed by 5 surgeons in 4 centers. No infection complication was observed other than 21 (2\%) wound leakage cases (5).

In conclusion, infectious complication is generally not observed in robotic thyroidectomy method.

\section{REFERENCES}

1. Lee KE, Choi JY, MD, Youn YK. Bilateral Axillo-Breast Approach Robotic Thyroidectomy. Surg Laparosc Endosc Percutan Tech 2011;21:230-6.

2. Tae $K$, Ji YB, Jeong JH, Lee SH, Jeong MA, Park CW. Robotic thyroidectomy by a gasless unilateral axillobreast or axillary approach: our early experiences. Surg Endosc 2011; 25:221-8.

3. Kim WW, Kim JS, Hur SM, et al. Is Robotic Surgery Superior to Endoscopic and Open Surgeries in Thyroid Cancer? World J Surg 2011; 35:779-84.

4. Lee KE, Koo do H, Kim SJ, et al. Outcomes of 109 patients with papillary thyroid carcinoma who underwent robotic total thyroidectomy with central node dissection via the bilateral axillo-breast approach. Surgery 2010;148:120713.

5. Lee J, Yun JH, Nam KH, Choi UJ, Chung WY, Soh EY. Perioperative clinical outcomes after robotic thyroidectomy for thyroid carcinoma: a multicenter study. Surg Endosc 2011;25:906-12.
${ }^{1}$ Pediatric Surgery, Children's Hospital, Gaziantep, ${ }^{2} \mathrm{Ksu}$ School of Medicine, Depar-
trment of Infection Disease, Kahramanmaras
Correspondence: Sevgi Büyükbese

Pediatric Surgery, Children's Hospital, Gaziantep, ${ }^{2} K s u$ School of Medicine, Departrment of Infection Disease, Kahramanmaras 